\title{
Lang wirksames Testosteron hilft hypogonadalen Männern
}

— „Die Testosterontherapie kann in großem Umfang die Abnahme des allgemeinen Wohlbefindens hypogonadaler Männer, die wir häufig mit dem Älterwerden verbinden, stoppen oder umkehren“, bestätigt Prof. Michael Zitzmann, Prüfleiter der IPASS (Internationalen Post-Authorisation Surveillance)-Studie.

Wie eine IPASS-Zwischenanalyse, deren Ergebnisse auf dem Weltkongress für Männergesundheit im Oktober 2008 in Wien präsentiert worden sind, bestätigt, verbessert das lang wirksame Testosteronundecanoat (Nebido ${ }^{\circledR}$ ) neben Libido und erektilen Funktionsstörungen auch Kraft, Vitalität, Stimmung und Konzent- rationsstörungen und vermindert Hitzewallungen und Schlafstörungen. Dies geht aus den Daten von 763 Männern hervor, die mindestens eine Injektion mit dem lang wirksamen Testosteronundecanoat erhalten hatten. Während der bis zu zwölfmonatigen Beobachtungszeit wurden keine schwerwiegenden behandlungsbezogenen Ereignisse und kein Fall von Prostatakarzinom dokumentiert. $88 \%$ der Behandelten zeigten sich mit ihrer Therapie „zufrieden“ oder „sehr zufrieden“. Die abschließenden Ergebnisse von IPASS werden im Lauf des Jahres 2010 erwartet.

dka

Nach Informationen von Bayer Healthcare

\section{Nykturie: Vasopressin-Analogon ist jetzt auch für Erwachsene verfügbar}

— Desmopressin, ein Analogon des antidiuretischen Hormons, wird von Kinderärzten seit vielen Jahren zur Therapie des Bettnässens eingesetzt. Seit Januar 2009 ist die Substanz als Nocturin ${ }^{\circledR}$ auch zur Behandlung der nächtlichen Polyurie bei Erwachsenen zugelassen.

Ursachen hierfür sind geschlechtsspezifisch unterschiedlich. „30\% der Männer mit benigner Prostatahypertrophie (BPH) müssen viermal pro Nacht zum Wasserlassen aufstehen, was zu erheblichen Folgestörungen wie Tagesmüdigkeit und Konzentrationsstörungen führt", erklärte

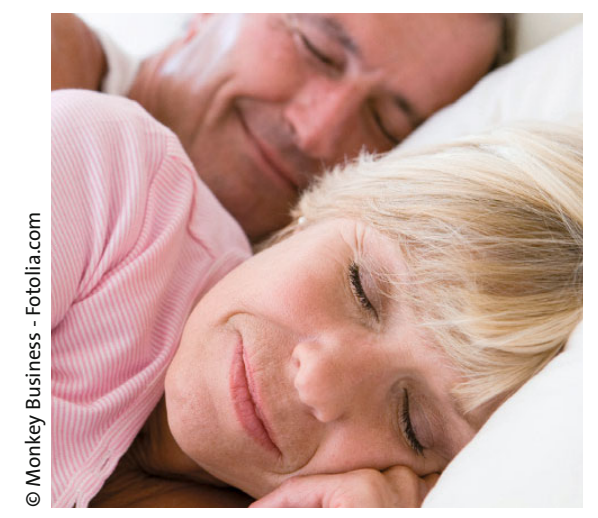

Erholsamer Schlaf ohne nächtlichen Gang zur Toilette.
Dr. Richard Berges, Köln. Bei Frauen ist die überaktive Blase häufigste Ursache einer Nykturie.

Das Vasopressin-Analogon Desmopressin erhöht in den distalen Nierentubuli und den Sammelröhren der Niere die Permeabilität für Wasser und verstärkt damit die Wasserrückresorption aus dem Primärharn. Dabei ist es deutlich besser verträglich als Vasopressin. Die antidiuretische Wirkung hält bis zu 14 Stunden lang an. Durch die Therapie kann eine Verlängerung der initialen Schlafdauer um mehr als zwei Stunden erreicht werden, die für den Erholungswert des Schlafs maßgeblich ist.

Wesentlich vor Therapiebeginn ist nach Ausführung von Berges eine exakte Diagnostik mit Hilfe eines Miktionsprotokolls. Die nächtliche Polyurie ist eine eigenständige Erkrankung mit Störung des normalen zirkadianen Arginin-Vasopressin-Rhythmus, die gegen andere Krankheitsbilder wie eine globale Polyurie abgegrenzt werden muss.

kam

Pressekonferenz „Ferring in der Urologie: Neue Maßstäbe, altersspezifische Therapiekonzepte“, 61. Kongress der Deutschen Gesellschaft für Urologie (DGU), Dresden, 18. September 2009; Veranstalter: Ferring

\section{Das Wandern wird des Mannes Lust}

WANDERN FÜR DIE MÄNNERGESUNDHEIT

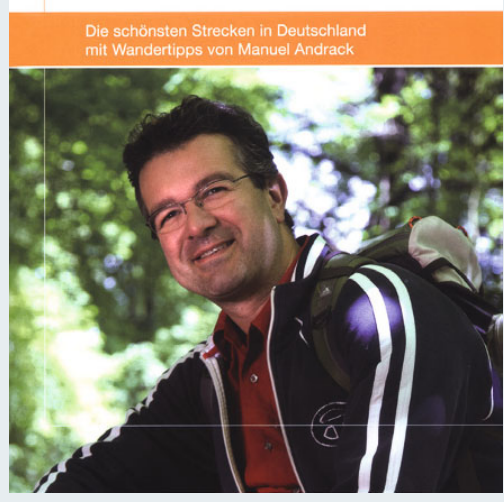

— Insbesondere Männer jenseits der 40 , also die „Best Ager“, profitieren von regelmäßiger körperlicher Bewegung - sie müssen es nur tun. Doch vier von fünf der Männer im besten Mannesalter treiben gar keinen oder nur wenig Sport. Übergewicht, Muskelabbau, Müdigkeit, Schlafstörungen, erektile Dysfunktion und Libidoverlust können die Folge sein.

Bayer Vital möchte Deutschlands Männer mit der Broschüre „Wandern für die Männergesundheit" wieder auf Trab bringen. Vorgestellt werden in dieser Broschüre einige der schönsten Wanderstrecken Deutschlands samt Tipps zum geeigneten Schuhwerk, zur Pauseneinteilung und dem richtigen Umgang mit Zecken.

dka

Ärzte und interessierte Patienten können die Broschüre entweder direkt aus dem Internet herunterladen (unter der Internetadresse www.testosteron.de) oder sie kostenlos bestellen bei:

Bayer Vital GmbH

Customer Service Center, Gebäude K56, 51368 Leverkusen

Tel. (01 80) 4574263

Fax (01 80) 4536274

E-Mail: maennergesundheit@bayerhealthcare.com. 